\title{
Antidiabéticos en Insuficiencia Cardíaca
}

\author{
Antidiabetics in Heart Failure \\ Antidiabéticos na Insuficiência Cardíaca
}

Zulay Andrea García Rojas ORCID: 0000-0001-6797-7308

Médico. Postgrado Medicina Interna Asistente interino Clínica Médica A Asistente de UMIC

Diana Marcela Cristancho

Sierra

ORCID: 0000-0001-6716-2264

Médico. Postgrado de Medicina

Interna. Asistente de UMIC

Amalia Verónica Pérez

Papadópulos

OR CID: 0000-0001-7892-995

Médico Internista. Prof. Adjunta

Clínica Médica A. Asistente de UMIC.

Gabriela Ormaechea

Gorricho

ORCID: 0000-0002-2981-7722

Médico Internista. Fellow de la ACP.

Prof. Directora Clínica Médica A.

Coordinadora de UMIC.
Resumen: Introducción. La insuficiencia cardíaca se reconoce como una enfermedad sistémica, por lo que se debe hacer un abordaje holístico y no enfocado exclusivamente a la falla cardíaca. La insuficiencia cardíaca se asocia con múltiples comorbilidades, siendo la diabetes mellitus una de las más frecuentes, estas comparten procesos fisiopatológicos con un comportamiento bidireccional, donde la mala evolución de una puede afectar a la otra. Por tanto, al considerar el tratamiento farmacológico de una de ellas, hay que tener en cuenta que el mismo no sea deletéreo para la otra. En los últimos años se requiere que cualquier tratamiento antidiabético tenga un efecto beneficioso o neutro a nivel cardiovascular. Este hecho es el constante desafío clínico al que el médico se enfrenta en estos pacientes. El objetivo de esta revisión es hacer una puesta a punto de la mejor evidencia disponible en el uso de los antidiabéticos en pacientes con insuficiencia cardíaca. Métodos. Se realizó una revisión sistemática de los principales estudios observacionales, ensayos clínicos, revisiones y metaanálisis publicados del uso de antidiabéticos y efecto cardiovascular, hasta diciembre del 2020, utilizando la base de datos de Pubmed y ScienceDirect. Conclusiones. De la revisión realizada se puede concluir que el fármaco de primera línea en pacientes con diabetes e insuficiencia cardíaca es la metformina, compartiendo este primer eslabón con los iSGLT2 (Empagliflozina, Canagliflozina y Dapagliflozina), según la última evidencia disponible, los que han demostrado ser eficaces en la reducción de las hospitalizaciones por insuficiencia cardíaca entre los pacientes con o sin diabetes y muerte cardiovascular, recientes estudios extienden además beneficio a los pacientes que también asocian enfermedad renal crónica.

Palabras clave: antidiabéticos, diabetes, insuficiencia cardiaca, iSGLT2, cardiovascular

Abstract: Introduction. Heart failure is recognized as a systemic disease, thus a holistic approach that is not exclusively focused on heart failure should be used. Heart failure is associated with multiple comorbidities, being diabetes mellitus one of the most frequent. These share pathophysiological processes with a bidirectional behavior, where the poor evolution of one can affect the other. Therefore, when considering the pharmacological treatment of one of them, it must be taken into account that it should not be detrimental to the other. In recent years, any antidiabetic treatment is required to have either a beneficial or a neutral effect at the cardiovascular level. This fact is the constant clinical challenge that physicians have to deal with when treating these patients. The objective of this review is to refine the best available evidence on the use of antidiabetic agents in patients with heart failure. Methods: A systematic review of the main observational studies, clinical trials, reviews and meta-analysis published on the use of antidiabetics agents and cardiovascular effect was carried out until December 2020, using Pubmed and ScienceDirect databases. Conclusions: From the review carried out, it can be concluded that the first-line drug for patients with diabetes and heart failure is metformin. This first link is shared with iSGLT2 (Empagliflozin, Canagliflozin and Dapagliflozin), according to the latest available evidence, which have been proven to be effective in reducing hospitalizations for heart failure among patients with or without diabetes and cardiovascular death. Recent studies also extend benefits to patients who are also associated with chronic kidney disease.

Key words: antidiabetic drugs, diabetes, heart failure, iSGLT2, cardiovascular

Resumo: Introdução. A insuficiência cardíaca é reconhecida como uma doença sistêmica, portanto, uma abordagem holística deve ser feita e não focada exclusivamente na insuficiência 
cardíaca. A insuficiência cardíaca está associada a múltiplas comorbidades, o diabetes mellitus uma das mais frequentes, pois compartilham processos fisiopatológicos com comportamento bidirecional, onde a má evolução de um pode afetar o outro. Portanto, ao se considerar o tratamento farmacológico de um deles, deve-se levar em consideração que não é prejudicial ao outro. Nos últimos anos, qualquer tratamento antidiabético é necessário para ter um efeito benéfico ou neutro no nível cardiovascular. Esse fato é o constante desafio clínico que o médico enfrenta nesses pacientes. O objetivo desta revisão é fazer uma pesquisa das melhores evidências disponíveis sobre o uso de anti-diabeticos em pacientes com insuficiência cardíaca. Métodos: Foi realizada uma revisão sistemática dos principais estudos observacionais, ensaios clínicos, revisões e metanálises sobre o uso de antidiabéticos e efeito cardiovascular, até dezembro de 2020, nas bases de dados Pubmed e ScienceDirect. Conclusões: A partir da revisão realizada, pode-se concluir que o medicamento de primeira linha em pacientes com diabetes e insuficiência cardíaca é a metformina, compartilhando esta primeira linha com o iSGLT2 (Empagliflozin, Canagliflozin e Dapagliflozin), de acordo com as últimas evidências disponíveis, para aqueles que se mostraram eficazes na redução de hospitalizações por insuficiência cardíaca entre pacientes com ou sem diabetes e morte cardiovascular, estudos recentes também estendem o benefício a pacientes que também associam doença renal crônica.

Palavras-chave: drogas antidiabéticas, diabetes, insuficiência cardíaca, iSGLT2, cardiovascular 


\section{Introducción}

La insuficiencia cardíaca (IC) es una enfermedad con alta y creciente prevalencia, morbimortalidad y elevados costos, representando un gran problema de salud pública a nivel mundial. Los esfuerzos por abatir estos desafíos son múltiples, con una búsqueda permanente de nuevas estrategias terapéuticas.

Es frecuente que los pacientes con IC asocien múltiples comorbilidades, las que deben ser correctamente diagnosticadas, evaluadas, y tratadas como parte del abordaje holístico de esta enfermedad. Existe un vínculo bidireccional entre la IC y cada una de las comorbilidades, donde la mala evolución de estas puede determinar un empeoramiento de la IC y viceversa. Dentro de las comorbilidades más frecuentes se destaca la diabetes mellitus (DM), la fibrilación auricular, los trastornos respiratorios del sueño, la ferropenia con o sin anemia, el hipotiroidismo y la enfermedad renal.

Por otro lado, más allá de considerar esta enfermedad como una mera asociación de patologías con evolución y pronóstico independientes, cada vez más se reconoce a la IC como una enfermedad sistémica. Por lo que el abordaje de la IC debe ser holístico y nunca enfocado exclusivamente a un concepto centrado en la falla cardíaca ${ }^{(1)}$.

Esta revisión se centrará en una de las comorbilidades más frecuentes en IC, como lo es la diabetes mellitus tipo 2 (DM2). La prevalencia de DM2 en pacientes con IC se encuentra entre

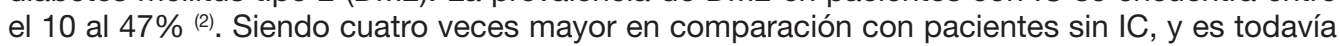
más alta en mayores de 60 años. No se puede olvidar que la DM2 es un factor de riesgo per se para IC.

Estudios observacionales han evidenciado un incremento de 2 a 4 veces el riesgo de IC en personas con DM2, en comparación con aquellos que no tienen dicha patología. El mal control glucémico se asocia con mayor riesgo de desarrollar IC, por cada aumento del $1 \%$ de la hemoglobina glicosilada ( $\mathrm{HbA1c}$ ) el riesgo de IC incrementa entre 8 y $36 \%$. Además, otros factores independientes como son el peso, dislipemia, obesidad, hipertensión arterial, nefropatía, enfermedad arterial, tiempo de evolución de la DM2 hacen que el riesgo de presentar IC se incremente. ${ }^{(2)}$

Los pacientes con IC que asocian DM2, tienen 75\% más riesgo de hospitalización y mortalidad, en comparación con los que no tienen la enfermedad. El aumento de $1 \mathrm{mmol} / \mathrm{L}$ de glucosa en ayunas se asocia con un incremento de 1,23 veces el riesgo de hospitalización por IC. ${ }^{(3)}$

A la luz de estos hallazgos se ha modificado el paradigma de estas patologías considerándose a ambas como enfermedades metabólicas, que comparten procesos fisiopatológicos, y que se comportan de manera bidireccional, es decir la mala evolución de una de las dos puede afectar a la otra.

Si se piensa entonces como patologías asociadas y vinculadas se deben abordar de manera conjunta, es decir al considerar el tratamiento farmacológico de una de ellas, se debe tener en cuenta no solo el beneficio, sino que el mismo no sea perjudicial para la otra enfermedad. Este hecho es el constante desafío clínico al que el médico se enfrenta en estos pacientes. Por lo que a la hora de elegir un antidiabético se debe considerar que el mismo tenga un efecto beneficioso o por lo menos neutro a nivel cardiovascular.

\section{Metodología}

Se analizaron las principales guías de manejo de Diabetes Mellitus tipo 2 en pacientes con insuficiencia cardíaca: Guía "Standard Medical Care in Diabetes 2020" y "Type 2 Diabetes Mellitus and Heart Failure A Scientific Statement From the American Heart Association and the Heart Failure Society of America".

Posteriormente se realizó una búsqueda utilizando la base de datos de Pubmed, se utilizaron las palabras claves: "antidiabetic treatment", "heart failure", el operador booleano "AND", uso de filtros (humanos y mayores de 19 años), se limitó a ensayos clínicos, revisiones y metaanálisis, desde el año 1998 hasta el 2020, encontrando 443 resultados. Además, en ScienceDirect se realizó la búsqueda con las palabras "diabetes" e "insuficiencia cardíaca" utilizando filtros para reducir la búsqueda (año 2020 y revista Colombiana de Cardiología), obteniendo 26 artículos.

Finalmente se eligieron artículos con acceso al texto completo y que fueran adecuados según el objetivo planteado. 


\section{Fisiopatología de la cardiomiopatía diabética}

Para comprender mejor de lo que se está hablando, al referirse a la cardiomiopatía diabética se realiza una breve reseña de su fisiopatología. Es así como fisiopatológicamente la IC se consideraba una enfermedad independiente de la DM. En el 2013, la Sociedad Europea de Cardiología y la Asociación Europea para el Estudio de la Diabetes, hacen énfasis en excluir otras causas etiológicas de la IC antes de concluir que esta sea secundaria exclusivamente por la DM. Otros autores sugieren que la cardiopatía diabética puede ocurrir de manera concomitante con otras enfermedades siendo la DM el mecanismo disparador de la etiología de la IC. (4-5)

La hipertrofia cardíaca en la DM es consecuencia del depósito de triglicéridos, del aumento del depósito de colágeno, de la fibrosis y de la hiperinsulinemia ocasionada por la resistencia a la insulina. ${ }^{(6-7)}$ Los productos avanzados de la glicosilación, constituyen un factor determinante para el daño microvascular en DM, causando modificación estructural y funcional cardíaca.

Es importante destacar que la aparición de la IC tiene un gran impacto en el metabolismo cardíaco, provocando un cambio en la utilización de sustratos cardíacos a partir de ácidos grasos y disminución de la oxidación de glucosa como sustrato energético. Este cambio incrementa el consumo de oxígeno, disminuye la actividad cardíaca y genera desacoplamiento mitocondrial, causando reducción de energía e induciendo estrés oxidativo, lo que genera alteración de la función del miocardio. ${ }^{(4,6)}$. La aparición de resistencia a la insulina en esta situación limita la oferta de energía y deteriora aún más la función cardíaca ${ }^{(7)}$. Varios estudios muestran que los pacientes con IC pueden presentar resistencia a la insulina, por múltiples mecanismos neurohumorales propios de esta patología, como son, el aumento de catecolaminas y cortisol e hipoperfusión sistémica. (7) La hipertrofia miocárdica produce un aumento de la fuerza contráctil para contrarrestar la tensión de la pared del ventrículo izquierdo (VI); ocasionando posteriormente dilatación ventricular izquierda, lo que lleva a una disminución en la fracción de eyección ventricular izquierda (FEVI). Por tanto, queda claro que un objetivo terapéutico en el tratamiento de la IC es lograr la reducción de tensión de la pared VI. ${ }^{(8)}$

En una publicación Seferovic y col ${ }^{(9)}$, describen dos fenotipos de la miocardiopatía diabética, siendo el primero el restrictivo, en el que predomina la remodelación concéntrica del VI y la disfunción diastólica VI. El segundo, el dilatado, presentado remodelación excéntrica y disfunción sistólica del VI. Es importante tener presente estos fenotipos, ya que conllevan diferentes tratamientos farmacológicos. En la figura 1, se resume de forma esquemática la fisiopatología de la cardiopatía diabética.
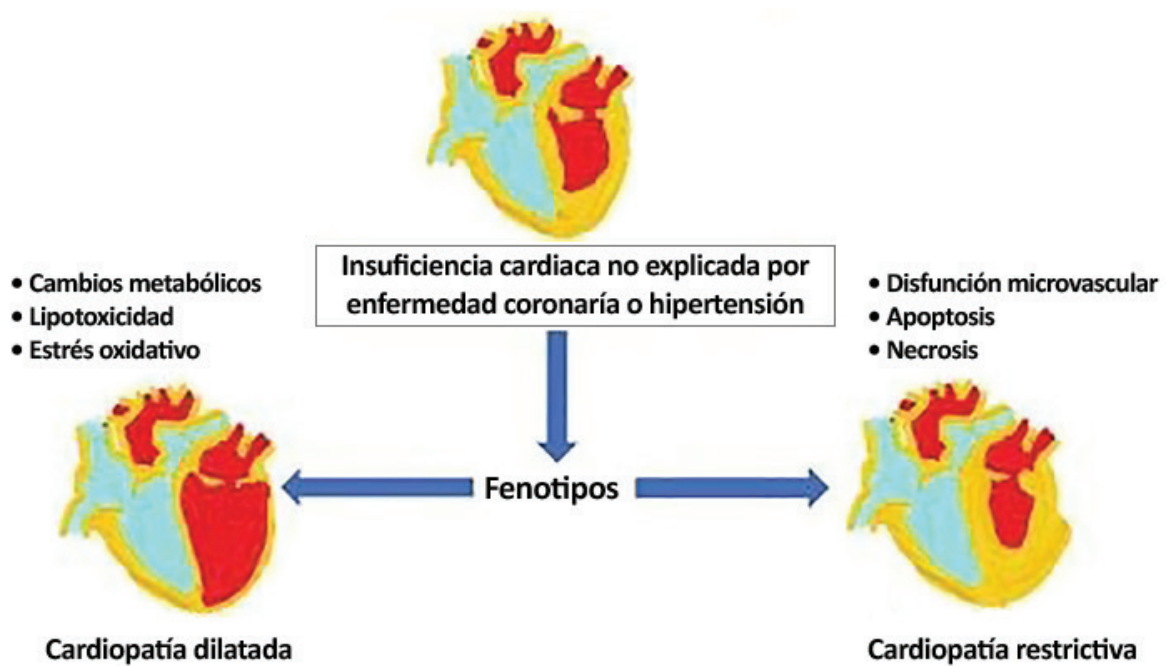


\section{Clasificación de la cardiomiopatía diabética}

La cardiomiopatía diabética se clasifica en 4 estadios. Esta clasificación utiliza parámetros estructurales cardíacos, clínicos, funcionales y paraclínicos, los cuales se describen en la tabla 1.

Tabla 1: Estadios de la cardiopatía diabética. Adaptado de la revista de la Sociedad Colombiana de Cardiología (4). Aceptado por la Dra. Clara Saldarriaga

\begin{tabular}{|l|l|l|l|l|}
\multicolumn{2}{|c}{ Clasificación } & \multicolumn{2}{c}{ Estadio 1 Estadio 2 } & \multicolumn{1}{c|}{ Estadio 3 } \\
Características & $\begin{array}{l}\text { Hipertrofia } \\
\text { ventricular }\end{array}$ & $\begin{array}{l}\text { Función ventricular } \\
\text { normal o reducida }\end{array}$ & $\begin{array}{l}\text { Función reducida, } \\
\text { microangiopatía }\end{array}$ & $\begin{array}{l}\text { Función } \\
\text { reducida, micro y } \\
\text { macroangiopatía }\end{array}$ \\
\hline Síntomas & $\begin{array}{l}\text { Con grandes } \\
\text { esfuerzos }\end{array}$ & $\begin{array}{l}\text { Con esfuerzos } \\
\text { moderados }\end{array}$ & $\begin{array}{l}\text { Con esfuerzos } \\
\text { pequeños }\end{array}$ & En reposo \\
\hline $\begin{array}{l}\text { Cambios } \\
\text { estructurales }\end{array}$ & $\begin{array}{l}\text { Aumento de la masa } \\
\text { ventricular }\end{array}$ & Dilatación y fibrosis & Dilatación y fibrosis & Dilatación y fibrosis \\
\hline $\begin{array}{l}\text { Cambios } \\
\text { funcionales }\end{array}$ & $\begin{array}{l}\text { Disfunción } \\
\text { diastólica }\end{array}$ & $\begin{array}{l}\text { Disfunción sistólica } \\
\text { y diastólica }\end{array}$ & $\begin{array}{l}\text { Disfunción sistólica y } \\
\text { diastólica }\end{array}$ & $\begin{array}{l}\text { Disfunción sistólica y } \\
\text { diastólica }\end{array}$ \\
\hline $\begin{array}{l}\text { Realce tardío en } \\
\text { resonancia cardíaca }\end{array}$ & Sin fibrosis & Posible fibrosis & Frecuente fibrosis & $\begin{array}{l}\text { Fibrosis muy } \\
\text { frecuentes }\end{array}$ \\
\hline $\begin{array}{l}\text { Biomarcadores } \\
\text { Troponina }\end{array}$ & Normales & Normales & $\begin{array}{l}\text { Elevados en caso } \\
\text { de inflamación 0 } \\
\text { isquemia }\end{array}$ & $\begin{array}{l}\text { Elevados en caso } \\
\text { de inflamación 0 } \\
\text { isquemia }\end{array}$ \\
\hline
\end{tabular}

Siguiendo el concepto de pensar a la IC como una enfermedad sistémica y a la hora de considerar el tratamiento farmacológico del paciente con IC y DM2, el médico se debería formular las siguientes preguntas: ¿todos los antidiabéticos se pueden prescribir en el paciente con IC?, ¿existe alguno más beneficioso que otro?, otro punto importante es el paciente con DM e IC que además asocia enfermedad renal, ¿se puede administrar cualquier antidiabético?

Esta revisión se abocará al tratamiento de la DM2 en pacientes con IC ya sea con FEVI preservada (FEVI mayor 50\%), intermedia (FEVI entre 40 y $50 \%$ ) o reducida ( $F E V I$ menor $40 \%$ ).

\section{Tratamiento farmacológico de la DM en el paciente con IC}

La estrategia óptima del tratamiento farmacológico en pacientes DM con IC sigue siendo controvertida. Algunos fármacos antidiabéticos tienen efectos deletéreos a nivel cardiovascular, es así como la FDA en el año $2008{ }^{(10)}$, publicó una guía dirigida a la industria farmacéutica en la que recomendó la evaluación del riesgo cardiovascular (CV) de los antidiabéticos que se habían lanzado recientemente al mercado y previo al lanzamiento de nuevos medicamentos para la diabetes. El criterio para la aceptación fue que los efectos no fuesen inferiores a los medicamentos ya existentes. (Estas recomendaciones fueron adoptadas por la Agencia Europea de medicamentos). Es decir, cualquier nuevo antidiabético debería cumplir con el hecho de tener por lo menos un efecto neutro o beneficioso a nivel CV. Por tanto, a la hora de tratar un paciente con DM2 e IC, no solo se debe pensar en el control metabólico, objetivo "glucocéntrico" de nuestros pacientes, sino que se debe pensar más en un objetivo centrado en el efecto CV, objetivo "cardiometabólico".

El estudio UKPDS (11) realizado en 1999 con 7600 participantes, en 23 centros del Reino Unido, tuvo como objetivo principal, determinar el efecto del control glucémico intensivo sobre la incidencia de complicaciones, el objetivo secundario fue evaluar si existían diferencias entre tratamientos antidiabéticos (sulfonilureas, insulina o metformina), asignados al azar para recibir terapia convencional o intensiva. Cuando la dieta no logró estos objetivos, los participantes fueron aleatorizados para recibir sulfonilureas, insulina o metformina, esta última solo en pacientes obesos. Frente al fracaso de los tratamientos individuales, se utilizaron combinaciones. EI UKPDS ${ }^{(11)}$ observó un efecto protector del control estricto de la glucosa sobre la enfermedad CV y la mortalidad general. A diferencia del UKPDS, el estudio ACCORD ${ }^{(12)}$, aleatorizado y controlado realizado en el 2008, que incluyó 10251 participantes tratados con insulina, sulfonilureas, metformina e incretinas, no observó ningún beneficio sobre la macrovasculatura con el control glucémico intensivo.

Un estudio de cohortes por Douglas y col (13), con 400.000 participantes, realizado con el objetivo de examinar la relación entre la HbA1c y la muerte por cualquier causa en pacientes con DM2 que posteriormente desarrollaron IC, mostró una menor mortalidad en aquellos pacientes que presentaban una $\mathrm{HbA} 1 \mathrm{c}$ entre 7 y $8 \%$, y se podría considerar valores entre $8-8,5 \%$ en paciente con expectativa de vida limitada, IC estadio D, enfermedad renal terminal o dependencia a oxígeno. 
Se concluiría de estos dos últimos estudios, que al tratar un paciente con DM, se debe individualizar el mismo, buscando objetivos de $\mathrm{HbA1C}$ diferenciales, teniendo en cuenta repercusiones de órgano blanco, comorbilidades, años de diabetes, edad del paciente y la expectativa de vida. Siendo más estricto en los pacientes más jóvenes y sin comorbilidades

\section{Fármacos antidiabéticos en IC}

Se podrían clasificar los fármacos antidiabéticos según el riesgo cardiovascular en 4 grupos. Las Sociedades Europea y Americana de Cardiología tienen claro su posicionamiento en relación con el riesgo cardiovascular de los fármacos antidiabéticos en paciente con IC, agrupándolos en:

1) Fármacos que aumentan el riesgo de IC: tiazolidinedionas y saxagliptina

2) Fármacos que pueden aumentar el riesgo de IC: sulfonilureas e insulina

3) Fármacos que se pueden usar con seguridad en IC: metformina, agonistas del receptor de GLP-1 (AR-GLP 1) y algunos Inhibidores de la dipeptidil peptidasa-4 (iDPP4), acarbosa

4) Fármacos que reducen el riesgo de IC: inhibidores del cotransportador sodio-glucosa tipo 2 (iSGLT2).

Se comentarán los mismos según familia de antidiabéticos.

\section{a) Sulfonillureas (SU)}

Las sulfonilureas estimulan la secreción de insulina a nivel pancreático, reduciendo los niveles de glucosa, con reducciones en ayuno del 36-72 mg/dl y HbA1c por $1 \%-2 \%$. Sin embargo, están asociadas con un riesgo significativo de hipoglucemia moderada en el $20 \%-40 \%$, y severa en 1\%-7\%. Pueden generar ganancia ponderal, su seguridad CV está cuestionada según diferentes estudios.

Claramente estos fármacos no tienen un efecto de clase en lo que refiere a su riesgo cardiovascular, siendo que algunos tienen efectos cardiovasculares deletéreos y otros neutros.

El ensayo UGDP ${ }^{(14)}$ informó un mayor riesgo de muerte CV asociada con el uso de tolbutamida en comparación con placebo o insulina, una justificación de la cardiotoxicidad inducida por las $\mathrm{SU}$ es el bloqueo del preacondicionamiento isquémico. El adelanto de nuevos medicamentos con sulfonilureas que no bloquean el preacondicionamiento isquémico ha originado que la controversia del UGDP sea discutible y ha preservado un lugar para las sulfonilureas en el tratamiento de la DM2. Todos estos efectos adversos se asocian fundamentalmente con las sulfonilureas de primera y algunas de segunda generación como la glibenclamida. Medicamentos más novedosos (por ej., la glimepirida) parecen no actuar en los canales cardíacos, razón por la cual implicaría un riesgo cardiovascular menor. Únicamente la gliclazida ha demostrado no aumentar las complicaciones macrovasculares y tener un efecto nefroprotector.

El estudio CAROLINA (15), que comparó la aparición de complicaciones CV en pacientes que recibían glimepirida vs linagliptina un inhibidor de DPP-4, evidenció un incremento de hipoglucemias con glimepirida, pero no demostró diferencias con respecto a la seguridad CV, ni de hospitalizaciones por IC.

Por tanto, hasta la fecha, las SU, como grupo no han demostrado reducir las complicaciones macrovasculares de la DM2, y parecen implicar un riesgo aumentado de eventos. Un beneficio que presentan estos medicamentos es su bajo costo.

Las SU deben ser usadas con precaución en pacientes con IC, ya que pueden aumentar el riesgo cardiovascular. No tiene un efecto de clase. Algunas SU cómo glimepirida y fundamentalmente gliclazida son las más aceptadas en pacientes con IC

\section{b) Biguanidas. Metformina: sensibilizadores}

La metformina se considera como el fármaco de elección en el manejo inicial de la DM2, ya sea en monoterapia o en terapia combinada. Con más de 50 años de experiencia en su uso, se considera que es el pilar fundamental del manejo de esta patología, siendo seguro, efectivo y de bajo costo.

Este medicamento tiene diversos mecanismos de acción, como son, reducción de la gluconeogénesis hepática y de la absorción intestinal de glucosa, por otro lado, aumenta la sensibilidad a la insulina en tejidos periféricos, además se cree que podría tener un rol protector 
en el endotelio vascular a través del incremento de óxido nítrico, contribuyendo a mejorar el estado protrombótico y proinflamatorio de las enfermedades cardiovasculares.

Por eso, a pesar de que se usa con precaución, se considera de acuerdo con la evidencia observacional que es un medicamento seguro, y además plantea ventajas respecto de otras intervenciones antidiabéticas.

Desde el estudio UKPDS ${ }^{(11)}$ realizado en 1999, se observó disminución del riesgo CV con reducción en la incidencia de infarto agudo de miocardio (IAM) y muerte coronaria en pacientes diabéticos tratados con metformina. Si bien la metformina se asoció con acidosis láctica en pacientes con insuficiencia renal concomitante, fue el único fármaco que demostró reducir la mortalidad por cualquier causa en pacientes con IC.

Un metaanálisis publicado en Circulation en el año 2013 que incluyó cerca de 3400 pacientes, mostró que la metformina estaba asociada con la reducción de la mortalidad $(0.80$ [95\% Cl, 0.74-0.87], y hospitalización por todas las causas $(0.93$ [95\% Cl, 0.89-0.98]) en pacientes con IC comparado con controles. ${ }^{(16)}$ En cuanto a sus efectos adversos, mucho se ha hablado de este medicamento y el riesgo de acidosis láctica, una revisión publicada en 2008 en Diabetes Care (17), sobre 347 ensayos clínicos y estudios de cohortes: no mostró una relación significativa entre el uso de metformina y acidosis láctica.

Estudios posteriores, como el de Gómez-Peralta y col ${ }^{(18)}$, pone de manifiesto la existencia de acidosis láctica por metformina fundamentalmente en pacientes octogenarios, con dosis plenas de metformina y en situaciones de insuficiencia renal aguda severa (creatinina media de 8), y siempre con Filtrados Glomerulares (FG) menores de $30 \mathrm{ml} / \mathrm{min}$. Por lo que se sugiere no iniciarla en pacientes con FG menor de $45 \mathrm{ml} / \mathrm{min}$ y contraindicarla en FG menores de $30 \mathrm{ml} / \mathrm{min}$.

La metformina es un fármaco seguro en pacientes diabéticos con IC o riesgo de $\mathrm{IC}$, en forma de monoterapia o asociado a otros antidiabéticos. Está contraindicada en pacientes con FG menor de $30 \mathrm{ml} / \mathrm{min}$, no se recomienda su inicio con FG menor de $45 \mathrm{ml} / \mathrm{min}$ y se sugiere la suspensión en condiciones agudas que se asocien con acidosis láctica, como shock cardiogénico y distributivo.

\section{c) Insulina}

Permanece como una terapia de segunda línea en DM2 con IC crónica, sin embargo, la seguridad de la insulina en este tipo de pacientes no se conoce hasta ahora. La insulina se relaciona con aumento de peso, retención de líquidos y riesgo de hipoglucemia, por lo que se debe emplear con moderación en pacientes con IC ${ }^{(19)}$. Un estudio de cohortes realizado por Gamble y col (20) donde el objetivo fue comparar las tasas de mortalidad por todas las causas y cardiovasculares en pacientes recién tratados con DM2 según la intensidad de exposición a insulina, incluyó 12272 participantes con terapia antidiabética oral entre 1991 y 1996 y se agruparon de acuerdo con la exposición acumulada de insulina basada en el total de dispensaciones de insulina por año: sin exposición (grupo de referencia); baja exposición (0 a $<3)$; exposición moderada (3 a <12) y exposición alta $(>12)$. Como resultado se observó que los pacientes que recibieron mayor cantidad de insulina presentaban mayor tasa de hospitalización por IC, mayor mortalidad, elementos de retención hidrosalina, elevación del NT-proBNP y requerían mayores dosis de furosemida. Estos resultados se podrían explicar en parte porque incluyeron pacientes diabéticos más graves o con enfermedad diabética más avanzadas ya que requirieron ser insulinizados.

Estudios más recientes como el ensayo clínico ORIGIN ${ }^{(21)}$ con 12612 participantes con riesgo alto de enfermedad CV con el uso de insulina glargina o tratamiento estándar (principalmente metformina y sulfonilureas), por un período de 6,2 años de seguimiento, no mostró diferencias en las hospitalizaciones por IC entre ambos grupos de tratamiento. También se observó un efecto neutro en infarto agudo de miocardio, accidente cerebro vascular, muerte cardiovascular y por todas las causas.

El estudio DEVOTE ${ }^{(22)}$ con 7637 pacientes, que comparó insulina Degludec vs. insulina Glargina, demostrando menor incidencia de hipoglucemia con insulina Degludec, no reveló diferencias respecto a la seguridad cardiovascular entre ambas insulinas.

Por tanto, se necesitan nuevos ensayos clínicos para definir el nivel de seguridad-riesgo del uso de insulina en estos pacientes 
La insulina puede aumentar el riesgo de IC o descompensar una IC. Se debe tener precaución al iniciar el tratamiento con insulina en pacientes diabéticos portadores de IC, de ser necesario se puede ajustar la dosis de diurético.

\section{d) Glitazonas (Tiazolidinedionas): Pioglitazona, rosiglitazona y troglitazona}

Estos medicamentos aumentan la sensibilidad a la insulina en el hígado y en el músculo esquelético, con poca o nula tendencia a producir hipoglucemia. Tienen acción antiinflamatoria y mejoran el perfil lipídico. Sin embargo, tanto la pioglitazona como la rosiglitazona incrementan la retención hídrica y sodio a nivel renal, lo que lleva a mayor riesgo de desarrollar o descompensar una IC. Estos efectos aumentan el riesgo de hospitalizaciones por IC, por lo que las guías Europeas en el 2016 contraindican su uso en estos pacientes en cualquier grado de IC (I al IV de la clasificación de la NYHA). ${ }^{(19)}$

La troglitazona se retiró debido a la hepatotoxicidad, las preocupaciones de seguridad sobre rosiglitazona y pioglitazona se elevó debido al aumento del riesgo cardiovascular (IAM e IC) y riesgo de cáncer de vejiga y fracturas óseas. ${ }^{(19)}$ En base a la preocupación de la seguridad CV, se realizó el ensayo RECORD ${ }^{(23)}$ estudio aleatorizado de 6 años de duración, con 4447 pacientes DM2 en monoterapia con metformina o sulfonilurea con $\mathrm{HbA} 1 \mathrm{c}$ media de $7,9 \%$ siendo asignados aleatoriamente con rosiglitazona $(n=2220)$ o combinación de metformina y sulfonilurea (grupo de control activo, $\mathrm{n}=2227$ ), con el objetivo de evaluar el impacto de la rosiglitazona a largo plazo de los efectos CV. El estudio confirmó que la adición de rosiglitazona a la terapia hipoglucemiante en personas con DM2 aumenta el riesgo de IC y de algunas fracturas, principalmente en mujeres. Aunque los datos no son concluyentes acerca de cualquier posible efecto sobre el infarto de miocardio, probablemente debido a que no era el objetivo a evaluar. Ante el riesgo cardiovascular evidenciado la Agencia Europea de medicamentos da la indicación de suspender la comercialización de la rosiglitazona.

Las glitazonas no están indicadas en pacientes diabéticos con IC por riesgo de incrementar retención hidrosalina lo que conduce a un aumento de eventos de descompensación de IC

\section{e) Agonistas del receptor de GLP 1 (AR-GLP 1)}

Este grupo de medicamentos imita la acción del péptido similar al glucagón (GLP 1). El GLP 1 se libera en el intestino ante la presencia de alimentos, incrementando la secreción de insulina por parte de las células $B$ pancreáticas.

Dentro de los efectos metabólicos se destacan: reducción de la $\mathrm{HbA1c}$ sin riesgo de hipoglucemia, dado que la secreción de insulina es directamente proporcional al de la glucosa, disminuye la secreción postprandial de glucagón en las células alfa pancreáticas, retrasa el vaciamiento gástrico con lo que genera un aumento de la saciedad y pérdida de peso estimada en 1-4 kg, estimulan la proliferación de células B e inhiben su apoptosis.

El ensayo clínico LEADER ${ }^{(24)}$ es un estudio doble ciego, que incluyó 9340 pacientes con DM2, comparó el efecto del liraglutide contra placebo en un seguimiento de 3.8 años; cuyo objetivo primario fue muerte cardiovascular, infarto de miocardio no fatal, ACV no fatal. Como resultado demostró superioridad del liraglutide, sin beneficios claros CV. El estudio ELIXA (25) con lixisenatide con 6068 participantes, diseñado para evaluar la seguridad y eficacia de ARGLP 1 en pacientes con DM2 y con riesgo de eventos CV. Donde el objetivo fue evaluar muerte cardiovascular, infarto miocardio no fatal, stroke no fatal y hospitalización. Lixisenatide demostró seguridad CV, pero no superioridad en la reducción de eventos CV. Algo similar se observó en los estudios PIONEER ${ }^{(25)}$, HARMONY TRIAL ${ }^{(26,27)}$, REWIND ${ }^{(28)}$ con semaglutide, albiglutide y dulaglutide, respectivamente.

Si bien se puede concluir que, hasta la fecha, los ensayos clínicos con AR-GLP 1, no han logrado demostrar un beneficio cardiovascular significativo en los pacientes diabéticos con IC, ni una disminución de hospitalización por IC, tampoco se ha demostrado un efecto deletéreo en estos pacientes; por lo que al momento se considera su uso como seguro. Otro hecho a considerar a la hora de prescribirlos es su alto costo y el uso subcutáneo.

Los agonistas del receptor GLP 1 son fármacos que pueden ser usados con seguridad en pacientes con DM e IC, si bien no se ha demostrado hasta el momento beneficios cardiovasculares 


\section{f) Inhibidores de la dipeptidil peptidasa-4 (iDPP-4)}

La DPP-4 es una enzima que degrada al péptido semejante al glucagón 1 (GLP 1) y polipéptido insulinotrópico dependiente de la glucosa (GIP). La inhibición de DPP-4 en la DM2 aumenta el nivel de GLP1 y GIP prolongando su actividad, generando un aumento de secreción de insulina y una reducción de la glucemia.

El estudio SAVOR TIMI $53^{(29)}$ con saxagliptina con 16492 pacientes con DM2 y enfermedad CV establecida o alto riesgo, observó un riesgo elevado de presentar IC en el seguimiento y un incremento del $27 \%$ en hospitalización por IC. El estudio EXAMINE ${ }^{(30)}$ incluyó 5380 pacientes postinfarto de miocardio que recibieron alogliptina vs placebo, también verificó aumento riesgo de IC y tasas elevadas de hospitalizaciones por IC. Por el contrario, el estudio TECOS ${ }^{(31)}$ con sitagliptina presentó resultados algo diferentes, en el que se incluyó 14671 pacientes con DM2 y enfermedad CV previa, 7332 pacientes en el grupo sitagliptina y 7339 en el grupo placebo. Los objetivos primarios de este estudio fueron compuestos por muerte cardiovascular, infarto de miocardio, ACV, ingreso por angina inestable e ingresos por IC. Los resultados han demostrado que con controles glucémicos similares no se observan diferencias en ninguno de los objetivos cardiovasculares definidos. Por tanto, se puede deducir que desde la perspectiva cardiovascular la sitagliptina es segura, no incrementa ingresos por IC y tiene un perfil global de seguridad muy favorable. No es inferior a la estrategia placebo desde la perspectiva cardiovascular.

El estudio CARMELINA ${ }^{(32)}$, realizado con el objetivo de evaluar el efecto de la linagliptina, sobre los resultados CV y los resultados renales en pacientes con DM2 con alto riesgo de eventos CV y renales. El end point primario fue el tiempo transcurrido hasta la primera aparición de la combinación de muerte CV, infarto de miocardio o accidente cerebrovascular no mortal, el secundario fue el tiempo transcurrido hasta la primera aparición de la muerte adjudicada a insuficiencia renal, enfermedad renal terminal o una disminución sostenida del $40 \%$ o más en la FG desde el inicio, otros objetivos fueron evaluar la tasa de hospitalizaciones por IC. Es un ensayo clínico, aleatorizado, multicéntrico, controlado con placebo, de no inferioridad realizado entre agosto de 2013 y agosto de 2016 en 605 centros en 27 países que incluyó 6979 pacientes con DM2, HbA1c 6,5\%-10,0\%, y alto riesgo CV, definido como enfermedad CV establecida y cociente albúmina/creatinina en orina (> $200 \mathrm{mg} / \mathrm{g}$ ), y/o alto riesgo renal ( $F G$ disminuido y/o micro o macroalbuminuria). Se excluyeron los pacientes con enfermedad renal crónica terminal. Este estudio concluyó que la linagliptina presenta un efecto neutro a nivel CV y cierto beneficio en hospitalización por IC.

Con estos estudios podemos concluir que los iDDP4 no tienen un efecto de clase, dado que algunos han mostrado ser deletéreos en lo CV, como se vio en el SAVOR TIMI con saxagliptina y EXAMINE con alogliptina, otros presentan un efecto neutro como TECOS y CARMELINA. Por tanto, estos fármacos han presentado una relación variable en la incidencia de IC, y hasta el momento no hay evidencia de un efecto beneficioso a nivel CV, por lo que no se recomienda su empleo como primera línea en diabéticos con IC o con riesgo de desarrollarla.

El metaanálisis de Zheng y col ${ }^{(33)}$, tuvo como objetivo comparar la eficacia de los inhibidores del cotransportador sodio-glucosa tipo 2 (iSGLT2), AR-GLP1 e iDPP4 sobre la mortalidad y los puntos finales cardiovasculares. Incluyó ensayos clínicos aleatorizados que reclutaron participantes con DM2 y un seguimiento de al menos 12 semanas. Se compararon iSGLT2, AR-GLP1 e iDPP4 entre sí o con placebo o sin tratamiento. Se concluyó que el uso de iSGLT2 o AR-GLP1 se asoció con una menor mortalidad que los iDPP-4 o placebo o ningún tratamiento. El uso de iDPP-4 se asociaron con $22 \%$ más riesgo de hospitalizaciones por IC en comparación con GLP-1, y $81 \%$ más riesgo en comparación con iSGLT2.

Los inhibidores de la DPP4 no deben ser la primera opción en pacientes con DM e IC. La mayoría tiene un efecto neutro cardiovascular. No presentan un efecto de clase, la Saxagliptina no se debería indicar en estos pacientes.

\section{g) Inhibidores del cotransportador sodio - glucosa tipo 2 (ISGLT2)}

Los iSGLT2 son una nueva familia de antidiabéticos orales que no solo han revolucionado el manejo de la DM2, sino también de varias patologías cardiovasculares entre ellas la IC. Aprobados por la FDA y la Agencia Europea de Medicamentos en el año 2014.

En condiciones normales, se estima que el $90 \%$ de la glucosa es reabsorbida en el túbulo proximal a través del cotransportador SGLT2, el $10 \%$ restante se reabsorbe en la parte más distal del túbulo proximal por el cotransportador SGLT1. Es de recalcar que el cotransportador SGLT2 puede estar sobre expresado en los pacientes con DM2, generando disminución de la glucosuria, incrementando la reabsorción de glucosa y por ende de la hiperglucemia. Al inhibir 
este cotransportador podría ocasionar mayor excreción de la glucosa a través de la orina y como consecuencia reducción de la glucemia. Lo anteriormente expuesto demuestra la importancia de esta nueva familia de antidiabéticos, ya que actúan inhibiendo el cotransportador de SGLT2 a nivel renal, por otro lado, también producen aumento de la excreción de sodio, generando reducción del volumen plasmático.

Su mecanismo de acción determina varios efectos metabólicos y cardiovasculares beneficiosos entre los que se destacan:

- Descenso de la presión arterial: Ensayos clínicos han mostrado la reducción de la presión arterial sistólica en promedio $3-5 \mathrm{mmHg}$ y la presión arterial diastólica en $2-3 \mathrm{mmHg}$, sin aumento compensatorio de la frecuencia cardíaca.

- Pérdida de peso secundaria a la glucosuria: Se estima pérdida de $180 \mathrm{~g}$ de glucosa, 240$400 \mathrm{Kcal} / \mathrm{d}$ ía con la administración crónica. Estudios observacionales han reportado descenso de 2-3 kg que ocurren fundamentalmente en los primeros meses de tratamiento. ${ }^{(34)}$

Estudios recientes, como el EMPAREG ${ }^{(35)}$, CANVAS ${ }^{(36)}$, DAPA-HF (37) realizado con iSGLT2; empagliflozina, canagliflozina, dapagliflozina respectivamente, han demostrado disminuir la mortalidad e ingresos hospitalarios por IC en pacientes diabéticos. Estos fármacos son los únicos antidiabéticos que han logrado un descenso significativo de las hospitalizaciones por IC.

El primer ensayo clínico controlado que reportó efectos benéficos a nivel cardiovascular con el uso de los iSGLT2 es el EMPAREG-OUTCOME ${ }^{(35)}$. Este estudio incluyó 7020 pacientes con DM2 y enfermedad CV establecida, los cuales fueron randomizados a placebo y empagliflozina con una dosis diaria de $10 \mathrm{mg}$ o $25 \mathrm{mg}$. Los resultados de este estudio mostraron una reducción del $38 \%$ de la mortalidad cardiovascular, 35\% en ingresos por IC descompensada a los 2 años y $32 \%$ en la mortalidad por cualquier causa.

Posteriormente dos estudios el CVD-REAL 1 y $2{ }^{(38-39)}$ con iSGLT2, basados en la práctica clínica real. EI CVD-REAL 1 analizó datos de 309.056 pacientes de 6 países (Japón, Corea del Sur, Singapur, Australia, Israel y Canadá) compararon pacientes que recién habían iniciado el tratamiento con cualquier iSGLT2 versus otros antidiabéticos en la práctica del mundo real y con diferentes iSGLT2, siendo el objetivo principal desarrollo de IC y muerte. Este estudio observó una reducción de $39 \%$ en IC, $51 \%$ para mortalidad de cualquier causa y $46 \%$ para reducción de IC o muerte, se concluyó que el tratamiento con iSGLT2 versus otros antidiabéticos presenta menor riesgo de IC congestiva y muerte.

En el estudio CVD-REAL $2{ }^{(39)}$, el objetivo fue evaluar resultados CV en pacientes que comenzaron con iSGLT2 versus otros antidiabéticos en regiones de Asia, Pacífico, Oriente Medio y América del Norte repitió la metodología del CVD-REAL 1, incluyó 235.064 pacientes en cada grupo. Observó que el uso de iSGLT2 frente a otros hipoglucemiantes se asoció con un menor riesgo de muerte (HR: 0,51; intervalo de confianza del 95\%: 0,37 a 0,70; $p<0,001)$, IC (HR: 0,64; intervalo de confianza del $95 \%$ : 0,50 a 0,82; $p=0,001)$, muerte o IC (HR: 0,60; intervalo de confianza 95\%: 0,47 a 0,76; $p<0,001$ ), infarto de miocardio (HR: 0,81; intervalo de confianza 95\%: 0,74 a 0,$88 ; p<0,001$ ) y accidente cerebrovascular (HR: 0,68; intervalo de confianza del 95\%: 0,55 a 0,84; $p<0,001$ ). Estos estudios sugieren que los beneficios observados con empagliflozina en un ensayo aleatorizado podrían ser aplicables a otros fármacos de su clase y a una amplia variedad de pacientes con DM2 en la práctica del mundo real.

El estudio CANVAS ${ }^{(36)}$, incluyó 10.142 participantes con DM2 y alto riesgo cardiovascular, fueron asignados al azar con canagliflozina versus placebo con un seguimiento de 188 semanas como media. El objetivo primario fue analizar la muerte cardiovascular y hospitalizaciones por IC. Se observó reducción en el riesgo de muerte cardiovascular, infarto de miocardio y stroke no fatales con canagliflozina (26.9 vs 31.5 por 1000 pacientes-año, HR 0.86; intervalo de confianza $95 \%$ 0.75-0.97). Además, una reducción en el riesgo de internación por IC (5.5 vs 8.7 cada 1000 pacientes/año, HR 0.67, intervalo de confianza 95\% 0.52-0.87). Sin embargo, se encontró un hallazgo no esperado de incremento en el riesgo de amputación en el grupo canagliflozina (6.3 vs 3.4 por 1000 pacientes -año, HR 1.97, intervalo de confianza 95\% 1.41-2.75). Aquellos pacientes con antecedente de IC previa, tuvieron mayor beneficio con este fármaco en lo que respecta a riesgo de muerte cardiovascular e internaciones por IC, en comparación con aquellos sin IC previa.

El estudio DECLARE-TIMI58 ${ }^{(40)}$, con dapagliflozina incluyó 17.160 participantes con DM2 y enfermedad cardiovascular establecida o múltiples factores de riesgo $\mathrm{CV}$. El objetivo principal de seguridad incluía los eventos cardiovasculares mayores (MACE: muerte cardiovascular, infarto e ictus). Los objetivos primarios de eficacia incluían el tiempo hasta la aparición de MACE y el tiempo hasta la aparición del compuesto de muerte cardiovascular y hospitalización por IC. 
Los objetivos secundarios de eficacia incluían un objetivo combinado renal (reducción $\geq 40 \%$ del FG, enfermedad renal terminal, muerte de causa renal o cardiovascular) y la mortalidad de cualquier causa. Los resultados de este ensayo mostraron que la dapagliflozina es superior al placebo para mejorar el control glucémico y no inferior pero no superior para reducir la MACE en pacientes con DM2 y alto riesgo CV. Hubo una reducción de las hospitalizaciones por IC y tuvieron menos probabilidades de sufrir desenlaces renales adversos y presentaron una reducción significativa en resultado cardiorrenal compuesto, tasas más altas de mejora en el cociente urinario de albúmina-creatinina que con el placebo.

Un metaanálisis de Zelniker y col (41), de ensayos publicados hasta el 24 de septiembre de 2018, con diferentes iSGLT2 vs placebo en pacientes con DM2 cuyos objetivos fueron repercusión CV, muerte y hospitalización por IC. Los objetivos de eficacia incluyeron eventos cardiovasculares adversos importantes (infarto de miocardio, accidente cerebrovascular o muerte cardiovascular), la combinación de muerte cardiovascular u hospitalización por IC y progresión de la enfermedad renal. En los resultados que se obtuvieron de este estudio, se observó con los iSGLT2 una reducción de los eventos cardiovasculares adversos mayores en un $11 \%$, del riesgo de muerte cardiovascular u hospitalización por IC en un $23 \%$, del riesgo de progresión de la enfermedad renal en un $45 \%$, con un beneficio similar en aquellos con y $\sin$ enfermedad cardiovascular aterosclerótica.

En el DAPA-HF ${ }^{(37)}$, ensayo controlado con placebo, se asignaron aleatoriamente 4.744 pacientes con IC con clase funcional II, III o IV de la New York Heart Association (NYHA) y una FEVI menor o igual a 40\%, en paciente con o sin DM2, para recibir dapagliflozina $10 \mathrm{mg}$ una vez al día o placebo, además de la terapia recomendada. El objetivo primario fue una combinación de empeoramiento de la IC (hospitalización o una consulta en emergencia que resultó en terapia intravenosa para la IC) o muerte CV. Se observó que el riesgo de empeoramiento de la IC o muerte por causas $\mathrm{CV}$ fue menor entre los que recibieron dapagliflozina con relación al placebo, independientemente de la presencia o ausencia de diabetes.

El estudio EMPEROR-Reducing ${ }^{(42)}$, ensayo aleatorizado y doble ciego para evaluar la eficacia y seguridad de empagliflozina $10 \mathrm{mg}$ una vez al día en comparación con placebo, en pacientes con IC crónica con FEVI reducida, en paciente con o sin DM2, el objetivo primario fue una combinación de muerte cardiovascular u hospitalización por empeoramiento de la IC. Incluyó 3730 participantes, durante una mediana de 16 meses. El número total de hospitalizaciones por IC fue menor en el grupo de empagliflozina. La tasa anual de disminución del FG fue más lenta en el grupo de empagliflozina que en el grupo de placebo $(-0,55$ frente a $-2,28 \mathrm{ml} / \mathrm{min} / 1,73 \mathrm{~m} 2$ del área de superficie corporal por año, $\mathrm{P}<0,001)$, y los pacientes tratados con Empagliflozina tenían un riesgo menor de resultados renales graves. Se concluyó que de los pacientes que recibieron la terapia recomendada para la IC, el grupo de empagliflozina tuvo un riesgo menor de muerte cardiovascular u hospitalización por IC que los del grupo de placebo, independientemente de la presencia o ausencia de diabetes.

Los iSGLT2 se consideran los fármacos de elección para el tratamiento de los pacientes con DM2 e IC, con impacto beneficioso a nivel CV y disminución de la mortalidad. Los iSGLT2 son los primeros antidiabéticos que han logrado disminuir las hospitalizaciones asociadas a IC.

La dapagliflozina y empagliflozina han demostrado ser efectivas en pacientes con insuficiencia cardíaca con fracción de eyección reducida, tanto en pacientes con o sin DM2.

\section{Tratamiento farmacológico de la DM2 e IC en el paciente con enfermedad renal crónica}

La presencia de DM2 e IC que además cursa con enfermedad renal crónica (ERC) es muy frecuente, hasta le fecha existen pocos estudios con relación a cuál sería el mejor tratamiento farmacológico cuando coinciden estas tres patologías.

Al momento de elegir el mejor antidiabético para estos pacientes diabéticos con IC y ERC, se debería considerar algunos aspectos:

- La metformina está contraindicada en paciente con FG menor a 30ml/min/1.73m2 dado el riesgo de acidosis láctica, por su eliminación renal se debe realizar ajuste según la función renal del paciente. No hay evidencia de que la metformina tenga un efecto a nivel de la progresión renal.

- Las SU también presentan eliminación renal, por lo que se debe ajustar la dosis según la función renal, en cuanto a la glimepirida se recomienda su uso en FG mayor a $60 \mathrm{ml} / \mathrm{min} / 1.73 \mathrm{~m} 2$, la gliclazida en FG mayor a $45 \mathrm{ml} / \mathrm{min} / 1.73 \mathrm{~m} 2$. 
- Los iDPP4 se pueden administrar en pacientes con enfermedad renal moderada a avanzada, la linagliptina al presentar metabolismo hepático no requiere ajuste de dosis, el resto de iDPP4 requieren ajuste de dosis según FG. Los IDPP4 no han demostrado reducción en progresión renal.

- En relación con los AR-GLP1 hay escasa evidencia de su uso en enfermedad renal avanzada, por lo que su indicación en pacientes con enfermedad renal estará guiada por su efecto a nivel ponderal, antidiabético y cardiovascular.

- En la ERC avanzada existe una disminución del aclaramiento de insulina lo que conduce a una mejoría en la tolerancia a la glucosa, pudiendo disminuir los requerimientos de insulina e incluso suspenderla. En estos pacientes tratados con insulina existe el doble de riesgo de padecer hipoglucemia grave, no se recomienda ajuste de dosis en FG mayor $50 \mathrm{ml} / \mathrm{min} / 1.73 \mathrm{~m} 2$. (43)

- Por último, con relación a los iSGLT2 se cuenta hasta el momento con dos estudios recientes que tienen como objetivo evaluar su efecto a nivel renal. Estos estudios se realizaron al observar que en los primeros ensayos clínicos con iSGLT2 existía además del beneficio cardiovascular, un efecto positivo a nivel renal.

Los estudios CANVAS ${ }^{(36)}$, EMPA-REG OUTCOME ${ }^{(35)}$ y DECLARE-TIMI $58{ }^{(40)}$, previamente comentados, ya mostraban en sus resultados exploratorios una mejora del pronóstico renal en DM2. Sin embargo, los resultados son discutidos dado que dichos estudios incluían a pacientes con ERC de bajo riesgo, y por otro lado en sus objetivos primarios no se incluía evaluar el impacto de estos fármacos a nivel renal. Los estudios CANVAS y EMPAREG-OUTCOME excluyeron pacientes con FG menor a $30 \mathrm{ml} / \mathrm{min} / 1.73 \mathrm{~m} 2$. El DECLARE-TIMI 58 tenía como criterio de inclusión una $F G \geq 60 \mathrm{ml} / \mathrm{min} / 1.73 \mathrm{~m} 2$, y el DAPA-HF ${ }^{(37)}$ incluyó pacientes con $\mathrm{FG}$ $\geq 30 \mathrm{ml} / \mathrm{min} / 1.73 \mathrm{~m} 2$.

El primer estudio que se realiza con el objetivo de evaluar los efectos de los iSGLT2 en pacientes con ERC y DM2 fue el ensayo clínico CREDENCE (44), doble ciego y aleatorizado, los pacientes con DM2 y ERC con albuminuria fueron asignados a recibir canagliflozina 100 $\mathrm{mg}$ al día o placebo. Los pacientes debían tener un $\mathrm{FG} \geq$ a $30 \mathrm{ml} / \mathrm{min} / 1,73 \mathrm{~m} 2$, albuminuria (cociente de albúmina/creatinina en orina de 300-5000/g) y recibir tratamiento con un inhibidor del sistema renina-angiotensina-aldosterona. El estudio se interrumpió de forma anticipada bajo recomendación del comité de seguimiento de datos debido a su eficacia. En ese momento incluía 4.401 pacientes aleatorizados, con tiempo medio de seguimiento de 2,26 años. El objetivo primario estaba compuesto por ERC en etapa terminal (diálisis, trasplante o un FG sostenida de $<15 \mathrm{ml} / \mathrm{min} / 1,73 \mathrm{~m} 2$ ), la duplicación del nivel de creatinina sérica o muerte de causa renal o cardiovascular. El riesgo relativo del objetivo compuesto de ERC terminal, duplicación del nivel de creatinina y muerte de causa renal se redujo un $34 \%$ y el riesgo relativo de ERC terminal se redujo un $32 \%$. También se evidenció un riesgo menor de muerte cardiovascular, infarto agudo de miocardio o stroke en el grupo de la canagliflozina (HR 0,80; IC 95\%: 0,67-0,95; $p=0,001$ ), así como una reducción de la hospitalización por IC (HR 0,61; IC 95\%: 0,47-0,80; p < 0,001). En conclusión, el riesgo de progresión de la enfermedad renal y eventos cardiovasculares fue menor en los pacientes DM2 con ERC que recibían tratamiento con canagliflozina frente a placebo.

El DAPA-CKD ${ }^{(45)}$ incluyó 4304 participantes con un FG estimada de 25 a $75 \mathrm{ml} / \mathrm{min} / 1,73$ m2 y una relación de albúmina - creatinina en orina 200 a $5000 \mathrm{~g}$, con dapagliflozina $10 \mathrm{mg}$ una vez al día o placebo. El objetivo primario fue combinado, disminución sostenida en el FG de al menos 50\%, enfermedad renal en etapa terminal o muerte por causas renales o cardiovasculares. El comité de seguimiento de datos recomendó detener el ensayo debido a su eficacia. Durante una mediana de 2,4 años. Se concluyó que entre los pacientes con ERC independientemente de la presencia o ausencia de DM, el riesgo de una disminución sostenida en el FG estimada de al menos 50\%, enfermedad renal en etapa terminal o muerte por causas renales o cardiovasculares fue significativamente menor con dapagliflozina que con placebo. Los efectos de la dapagliflozina fueron similares en los participantes con DM2 y en aquellos sin diabetes tipo 2. Se confirmó el perfil de seguridad conocido de dapagliflozina.

Actualmente existe un estudio en curso, el EMPA-KIDNEY ${ }^{(46)}$, un ensayo clínico multicéntrico, aleatorizado, doble ciego, controlado con placebo, de empagliflozina una vez al día, para evaluar los resultados cardiorenales en pacientes con ERC, que incluye pacientes con $F G \geq 20 \mathrm{ml} / \mathrm{min}$, tener dosis apropiadas IECA o ARAIl, a menos que dicho tratamiento no sea tolerado o no esté indicado. El objetivo principal del estudio es investigar el efecto de la empagliflozina sobre la progresión de la enfermedad renal y la muerte cardiovascular frente a placebo. 
No hay suficiente evidencia del mejor tratamiento antidiabético de pacientes con IC- DM y ERC.

- La metformina se puede administrar en FG mayores a $30 \mathrm{ml} / \mathrm{min} / 1.73 \mathrm{~m} 2$

- La glimepirida se recomienda su uso en FG mayor a $60 \mathrm{ml} / \mathrm{min} / 1.73 \mathrm{~m} 2$ y la gliclazida en FG mayor a $45 \mathrm{ml} / \mathrm{min} / 1.73 \mathrm{~m} 2$

- La linagliptina no requiere ajuste de dosis, el resto de iDPP4 requieren ajuste de dosis según FG

- Los AR-GLP1 tienen escasa evidencia de su uso en enfermedad renal avanzada

- Con la insulina no se recomienda ajuste de dosis en FG mayor $50 \mathrm{ml} / \mathrm{min} / 1.73 \mathrm{~m} 2$

- La canagliflozina y dapagliflozina han demostrado tener beneficio a nivel renal.

En la tabla 2 se resume los diferentes grupos de antidiabéticos con sus efectos a nivel cardiovascular y posología en enfermedad renal crónica.

\begin{tabular}{|c|c|c|c|c|c|}
\hline \multirow{2}{*}{$\begin{array}{c}\text { Grupo } \\
\text { farmacológico }\end{array}$} & \multirow[b]{2}{*}{ Antidiabético } & \multicolumn{3}{|c|}{ Efecto a nivel cardiovascular } & \multirow{2}{*}{ Posología en enfermedad renal } \\
\hline & & Beneficio & Neutro & Riesgo & \\
\hline Biguanidas & Metformina & $x$ & & & $\begin{array}{l}\text { FG > 60: } 500-1000 \mathrm{mg} / 8-12 \mathrm{~h} \\
\text { FG 45-60: Máx. } 2000 \mathrm{mg} / 24 \mathrm{~h} \\
\text { FG 30-45: Máx } 1000 \mathrm{mg} / 24 \mathrm{~h} \\
\text { FG <30 y diálisis: contraindicado }\end{array}$ \\
\hline \multirow{4}{*}{$\begin{array}{l}\text { Inhibidores } \\
\text { SGLT-2 }\end{array}$} & Empagliflozina & $x$ & & & $\begin{array}{l}\mathrm{FG}>60: 10-25 \mathrm{mg} / 24 \mathrm{~h} \\
\mathrm{FG} 30-60: \text { Mantener si } \\
\text { prescrito, } 10 \mathrm{mg} / 24 \mathrm{~h} \\
\mathrm{FG}<30 \text { : contraindicado }\end{array}$ \\
\hline & Canagliflozina & $x$ & & & $\begin{array}{l}\text { FG > 60: } 100-300 \mathrm{mg} / 24 \mathrm{~h} \\
\mathrm{FG} 30-60 \text { : mantener } 100 \mathrm{mg} / 24 \mathrm{~h} \\
\mathrm{FG}<30 \text { : contraindicado }\end{array}$ \\
\hline & Dapagliflozina & & $x$ & & $\begin{array}{l}\mathrm{FG}>60: 10 \mathrm{mg} / 24 \mathrm{~h} \\
\mathrm{FG} \mathrm{45-60:} \mathrm{mantener} \\
\mathrm{FG} \mathrm{45-25:} \mathrm{mantener} \\
\mathrm{FG}<25 \text { : contraindicado }\end{array}$ \\
\hline & Ertugliflozina & & $x$ & & $\begin{array}{l}\mathbf{F G}>60: 5-15 \mathrm{mg} / 24 \mathrm{~h} \\
\mathrm{FG} 45-60: \text { mantener si prescrito } \\
\mathrm{FG}<45: \text { contraindicado }\end{array}$ \\
\hline
\end{tabular}




\begin{tabular}{|c|c|c|c|c|}
\hline \multirow{4}{*}{$\begin{array}{l}\text { Agonistas } \\
\text { GLP-1 }\end{array}$} & Liraglutide & $x$ & & $\begin{array}{l}\mathrm{FG}>60: 1,2-1,8 \mathrm{mg} / 24 \mathrm{~h} \\
\mathrm{FG} 45-60: 1,2-1,8 \mathrm{mg} / 24 \mathrm{~h} \\
\mathrm{FG} 30-45: 1,2-1,8 \mathrm{mg} / 24 \mathrm{~h} \\
\mathrm{FG} 15-30: 1,2-1,8 \mathrm{mg} / 24 \mathrm{~h} \\
\mathrm{FG}<15: 1,2-1,8 \mathrm{mg} / 24 \mathrm{~h} \\
\text { Diálisis: no se recomienda }\end{array}$ \\
\hline & Semaglutide & $x$ & & 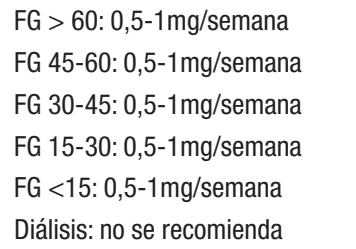 \\
\hline & Exenatide & $x$ & & $\begin{array}{l}\text { FG > 60: } 2 \mathrm{mg} / \text { semana } \\
\text { FG 45-60: } 2 \mathrm{mg} / \text { semana } \\
\text { FG 30-45: } 2 \mathrm{mg} / \text { semana } \\
\text { FG 15-30: no se recomienda } \\
\text { FG <15: no se recomienda } \\
\text { Diálisis: no se recomienda }\end{array}$ \\
\hline & Lixisenatide & & $x$ & $\begin{array}{l}\text { FG > 60: } 20 \mathrm{mcg} / 24 \mathrm{~h} \\
\text { FG 45-60: } 20 \mathrm{mcg} / 24 \mathrm{~h} \\
\text { FG 30-45: } 20 \mathrm{mcg} / 24 \mathrm{~h} \\
\text { FG 15-30: no se recomienda } \\
\text { FG <15: no se recomienda } \\
\text { Diálisis: no se recomienda }\end{array}$ \\
\hline \multirow{4}{*}{$\begin{array}{l}\text { Inhibidores } \\
\text { DPP-4 }\end{array}$} & Linagliptina & & $x$ & $\begin{array}{l}\mathrm{FG}>60: 5 \mathrm{mg} / 24 \mathrm{~h} \\
\mathrm{FG} 45-60: 5 \mathrm{mg} / 24 \mathrm{~h} \\
\mathrm{FG} 30-45: 5 \mathrm{mg} / 24 \mathrm{~h} \\
\mathrm{FG}<30 \text { y diálisis: } 5 \mathrm{mg} / 24 \mathrm{~h}\end{array}$ \\
\hline & Saxagliptina & & & $\begin{array}{l}\mathrm{FG}>60: 5 \mathrm{mg} / 24 \mathrm{~h} \\
\mathrm{FG} 45-60: 5 \mathrm{mg} / 24 \mathrm{~h} \\
\mathrm{FG} 30-45: 2,5 \mathrm{mg} / 24 \mathrm{~h} \\
\mathrm{FG}<30: 2,5 \mathrm{mg} / 24 \mathrm{~h} \\
\text { Diálisis: no recomendado }\end{array}$ \\
\hline & Sitagliptina & & $x$ & $\begin{array}{l}\mathrm{FG}>60: 100 \mathrm{mg} / 24 \mathrm{~h} \\
\mathrm{FG} 45-60: 100 \mathrm{mg} / 24 \mathrm{~h} \\
\mathrm{FG} \mathrm{30-45:} 50 \mathrm{mg} / 24 \mathrm{~h} \\
\mathrm{FG}<30: 2,5 \mathrm{mg} / 24 \mathrm{~h} \\
\text { Diálisis: } 2,5 \mathrm{mg} / 24 \mathrm{~h}\end{array}$ \\
\hline & Vildagliptina & & $x$ & $\begin{array}{l}\mathrm{FG}>60: 50 \mathrm{mg} / 12 \mathrm{~h} \\
\mathrm{FG} 45-60: 50 \mathrm{mg} / 12 \mathrm{~h} \\
\mathrm{FG} 30-45: 50 \mathrm{mg} / 24 \mathrm{~h} \\
\mathrm{FG}<30: 50 \mathrm{mg} / 24 \mathrm{~h} \\
\text { Diálisis: } 50 \mathrm{mg} / 24 \mathrm{~h}\end{array}$ \\
\hline \multirow{4}{*}{ Sulfonilureas } & Glibenclamida & & & No se recomienda \\
\hline & Glimepirida & & $x$ & $\begin{array}{l}\text { Usar si FG > 60: } 1-4 \mathrm{mg} / 24 \mathrm{~h} \\
\mathrm{FG} 45-60 \text { : precaución } \\
\mathrm{FG}<45 \text { y diálisis: contraindicado }\end{array}$ \\
\hline & Glicazida & & $x$ & $\begin{array}{l}\mathrm{FG}>60: 30-120 \mathrm{mg} / 24 \mathrm{~h} \\
\mathrm{FG} 30-60 \text { : precaución } \\
\mathrm{FG}<30 \text { y diálisis: contraindicado }\end{array}$ \\
\hline & Glipizida & & $x$ & $\begin{array}{l}\mathrm{FG}>60: 5-15 \mathrm{mg} / 12-24 \mathrm{~h} \\
\mathrm{FG} 45-60 \text { : precaución } \\
\mathrm{FG}<45 \text { y diálisis: contraindicado }\end{array}$ \\
\hline
\end{tabular}


Tabla 2: Diferentes grupos de hipoglicemiantes y sus efectos a nivel cardiovascular y posología en enfermedad renal crónica

\begin{tabular}{|c|c|c|c|}
\hline \multirow{2}{*}{ Insulinas } & Humanas & $x$ & $\begin{array}{l}\text { No ajuste de dosis si FG }>50 \\
\text { FG 10-50: dosis inicial un } 75 \% \text { (7U) }\end{array}$ \\
\hline & Análogos & $x$ & $\begin{array}{l}\text { No ajuste de dosis si FG }>50 \\
\text { FG 10-50: dosis inicial un } 75 \% \text { (7U) }\end{array}$ \\
\hline Glitazonas & $\begin{array}{l}\text { Rosiglitazona } \\
\text { Pioglitazona }\end{array}$ & & $\begin{array}{l}\mathrm{FG}>60: 15-45 \mathrm{mg} / 24 \mathrm{~h} \\
\mathrm{FG} 30-60 \text { : precaución } \\
\mathrm{FG}<30 \text { : precaución } \\
\text { Diálisis: contraindicado }\end{array}$ \\
\hline
\end{tabular}

\section{Conclusiones}

La prevalencia de insuficiencia cardíaca entre los pacientes diabéticos es cuatro veces más alta que en la población general. Este binomio IC-DM les confiere a los pacientes mayor mortalidad.

Los objetivos de la hemoglobina glicosilada se deben manejar de manera individualizada, según las características de cada paciente. El tratamiento de estos pacientes no debe ser con un objetivo glucocéntrico sino cardiometabólico. Es decir, al realizar el tratamiento farmacológico de la diabetes en paciente con insuficiencia cardíaca, se debe tener presente el efecto de estos medicamentos a nivel cardiovascular, ya que algunos antidiabéticos tienen efectos cardiovasculares negativos. Se debe utilizar aquellos con efecto beneficioso a nivel cardiovascular o comportamiento neutro.

El fármaco de primera línea en el paciente con diabetes e insuficiencia cardíaca es la metformina, compartiendo este primer eslabón con los iSGLT2 (empagliflozina, canagliflozina y dapagliflozina), según la última evidencia, los que han demostrado ser eficaces en la reducción de las hospitalizaciones por insuficiencia cardíaca en pacientes con o sin diabetes, recientes estudios extienden además beneficio a los pacientes que asocian enfermedad renal crónica. Por lo que se puede decir que los iSGLT2 se están ubicando como fármacos cardioprotectores y nefroprotectores, con evidencia para tratar la ERC y la IC con fracción de eyección reducida, independiente de la presencia de diabetes.

\section{Conflicto de interés}

Los autores declaran no tener ningún conflicto de interés.

\section{Bibliografía}

1- Saldarriaga-Giraldo C. Prólogo - Insuficiencia cardíaca y diabetes mellitus: Las dos caras de la moneda. Rev Colomb Cardiol. 2020; 27 (S2):2. https://doi.org/10.1016/j.rccar.2020.01.003.

2- Dunlay SM, Givertz MM, Aguilar D, Allen LA, Chan M, Desai AS, et al. Type 2 Diabetes Mellitus and Heart Failure: A Scientific Statement From the American Heart Association and the Heart Failure Society of America: This statement does not represent an update of the 2017 ACC/AHA HFSA heart failure guideline update. Circulation. 2019 Aug 13;140 (7):e294-e324. doi: 10.1161/ CIR.0000000000000691.

3- González-Robledo G, Jaramillo Jaramillo M, Comín-Colet J. Diabetes mellitus, insuficiencia cardíaca y enfermedad renal crónica. Rev Colomb Cardiol. 2020; 27 (S2): 3-6.

4- Saldarriaga-Giraldo C, Navas V, Morales C. De la diabetes a la insuficiencia cardíaca ¿Existe la miocardiopatía diabética? Rev Colomb Cardiol. 2020; 27 (S2): 12-16. DOI: 10.1016/j.rccar.2019.12.006

5- Borghetti G, von Lewinski D, Eaton DM, Sourij H, Houser SR, Wallner M. Diabetic Cardiomyopathy: Current and Future Therapies. Beyond Glycemic Control. Front Physiol. 2018 Oct 30;9:1514. doi: 10.3389/fphys.2018.01514.

6- Isfort M, Stevens SCW, Schaffer S, Ju Jong C, Wold LE. Disfunción metabólica en la miocardiopatía diabética. Heart Fail Rev. 2014;19, 35-48. https://doi.org/10.1007/s10741-013-9377-8.

7- Kostis JB, Sanders M. The association of heart failure with insulin resistance and the development of type 2 diabetes. Am J Hypertens. 2005 May; 18(5 Pt 1):731-7. doi: 10.1016/j.amjhyper.2004.11.038.

8- Rodríguez-González MJ, Contreras J. De la insuficiencia cardíaca a la diabetes. ¿Existe un mecanismo común? Rev Colomb Cardiol. 2020; 27 (S2): 7-11. 
9- Seferović PM, Paulus WJ. Clinical diabetic cardiomyopathy: a two-faced disease with restrictive and dilated phenotypes. Eur Heart J. 2015 Jul 14;36(27):1718-27, 1727a-1727c. doi: 10.1093/eurheartj/ ehv134.

10- Food and Drug Administration.FDA news release: FDA Announces new recommendations on evaluating cardiovascular risk in drugs intended to treat Type 2 diabetes.December 17, 2008. Silver Spring, MD: FDA, 2008.

11- King P, Peacock I, Donnelly R. The UK prospective diabetes study (UKPDS): clinical and therapeutic implications for type 2 diabetes. Br J Clin Pharmacol. 1999 Nov;48(5):643-8. doi: 10.1046/j.13652125.1999.00092.x

12- Dluhy RG, McMahon GT. Intensive glycemic control in the ACCORD and ADVANCE trials. N Engl J Med. 2008 Jun 12;358(24):2630-3. doi: 10.1056/NEJMe0804182.

13- Elder DH, Singh JS, Levin D, Donnelly LA, Choy AM, George J, et al. Mean HbA1c and mortality in diabetic individuals with heart failure: a population cohort study. Eur J Heart Fail. 2016 Jan;18(1):94102. doi: 10.1002/ejhf.455

14- Simpson SH, Majumdar SR, Tsuyuki RT, Eurich DT, Johnson JA. Dose-response relation between sulfonylurea drugs and mortality in type 2 diabetes mellitus: a population-based cohort study. CMAJ. 2006 Jan 17;174(2):169-74. doi: 10.1503/cmaj.050748.

15- Rosenstock J, Kahn SE, Johansen OE, Zinman B, Espeland MA, Woerle HJ, et al. Effect of Linagliptin vs Glimepiride on Major Adverse Cardiovascular Outcomes in Patients With Type 2 Diabetes: The CAROLINA Randomized Clinical Trial. JAMA. 2019 Sep 19;322(12):1155-1166. doi: 10.1001/ jama.2019.13772.

16- Shah DD, Fonarow GC, Horwich TB. Metformin therapy and outcomes in patients with advanced systolic heart failure and diabetes. J Card Fail. 2010 Mar;16(3):200-6. doi: 10.1016/j. cardfail.2009.10.022

17- Bodmer M, Meier C, Krähenbühl S, Jick SS, Meier CR. Metformin, sulfonylureas, or other antidiabetes drugs and the risk of lactic acidosis or hypoglycemia: a nested case-control analysis. Diabetes Care. 2008 Nov;31(11):2086-91. doi: 10.2337/dc08-1171.

18- Gomez-Peralta F, Escalada San Martín FJ, Menéndez Torre E, Mata Cases M, Ferrer García JC, Ezkurra Loiola P, et al. Recomendaciones de la Sociedad Espanola de Diabetes (SED) para el tratamiento farmacológico de la hiperglucemia en la diabetes tipo 2: Actualización 2018. Endocrinol Diabetes Nutr. 2018;65:611

19- Amed Castillo G, Morales-Villegas E. Tratamiento de la diabetes en el paciente con insuficiencia cardíaca: Más allá de la hemoglobina glicosilada. Rev Colomb Cardiol. 2020; 27(S2): 33-39.

20- Gamble JM, Simpson SH, Eurich DT, Majumdar SR, Johnson JA. Insulin use and increased risk of mortality in type 2 diabetes: a cohort study. Diabetes Obes Metab. 2010 Jan;12(1):47-53. doi: 10.1111/j.1463-1326.2009.01125.x.

21- Gerstein H, Yusuf S, Riddle MC, Ryden L, Bosch J. Rationale, design, and baseline characteristics for a large international trial of cardiovascular disease prevention in people with dysglycemia: The ORIGIN Trial (Outcome Reduction with an Initial Glargine Intervention). Am Heart J 2008; 155:26-32

22- Marso SP, McGuire DK, Zinman B, Poulter NR, Emerson SS, Pieber TR, et al. Efficacy and Safety of Degludec versus Glargine in Type 2 Diabetes. N Engl J Med 2017 Aug 24;377(8):723-732. doi: 10.1056/ NEJMoa1615692. Epub 2017 Jun 12.

23- Home PD, Pocock SJ, Beck-Nielsen H, Curtis PS, Gomis R, Hanefeld M, et al. Rosiglitazone evaluated for cardiovascular outcomes in oral agent combination therapy for type 2 diabetes (RECORD): a multicentre, randomised, open-label trial. Lancet 2009; 373:2125-35.

24- Marso SP, Daniels GH, Brown-Frandsen K, Kristensen P, Mann JFE, Nauck MH, et al. Liraglutide and Cardiovascular Outcomes in Type 2 Diabetes. N Engl J Med. 2016 Jul 28;375(4):311-22. doi: 10.1056/ NEJMoa1603827.

25- Pfeffer MA, Claggett B, Diaz R, Dickstein K, Gerstein HC, Køber LV, et al. Lixisenatide in Patients with Type 2 Diabetes and Acute Coronary Syndrome. N Engl J Med 2015; 373:2247-2257 DOI: 10.1056/ NEJMoa1509225

26- Husain M, Birkenfeld AL, Donsmark M, Dungan K, Eliaschewitz FG, Franco DR, et al. Oral Semaglutide and Cardiovascular Outcomes in Patients with Type 2 Diabetes. N Engl J Med. 2019 Aug 29;381(9):841-851. doi: 10.1056/NEJMoa1901118.

27- Hernandez AF, Green JB, Janmohamed S, D’Agostino RB Sr, Granger CB, Jones NP, et al. Albiglutide and cardiovascular outcomes in patients with type 2 diabetes and cardiovascular disease 
(Harmony Outcomes): a double-blind, randomised placebo-controlled trial. Lancet. 2018 Oct 27;392(10157):1519-1529. doi: 10.1016/S0140-6736(18)32261-X.

28- Gerstein HC, Colhoun HM, Dagenais GR, Diaz R, Lakshmanan M, Pais P, et al. Dulaglutide and cardiovascular outcomes in type 2 diabetes (REWIND): a double-blind, randomised placebo-controlled trial. Lancet. 2019 Jul 13;394(10193):121-130. doi: 10.1016/S0140-6736(19)31149-3.

29- Scirica BM, Braunwald E, Raz I, Cavender MA, Morrow DA, Jarolim P, et al. Heart failure, saxagliptin, and diabetes mellitus: observations from the SAVOR-TIMI 53 randomized trial. Circulation 2014 Oct 28;130(18):1579-88. doi: 10.1161/CIRCULATIONAHA.114.010389.

30- White WB, Cannon CP, Heller SR, Nissen S, Bergenstal RM, Bakris GL, et al. Alogliptin after Acute Coronary Syndrome in Patients with Type 2 Diabetes. N Engl J Med 2013; 369:1327-1335. DOI: 10.1056/NEJMoa1305889

31- Green JB, Bethel MA, Armstrong PW, Buse JB, Engel SS, Garg J, Josse R, et al. Effect of Sitagliptin on Cardiovascular Outcomes in Type 2 Diabetes. N Engl J Med. 2015 Jul 16;373(3):232-42. doi: 10.1056/ NEJMoa1501352.

32- Rosenstock J, Perkovic V, Johansen OE, Cooper ME, Kahn SE, Marx N, et al. Effect of Linagliptin vs Placebo on Major Cardiovascular Events in Adults With Type 2 Diabetes and High Cardiovascular and Renal Risk: The CARMELINA Randomized Clinical Trial. JAMA. 2019 Jan 1;321(1):69-79. doi: 10.1001/ jama.2018.18269.

33- Zheng SL, Roddick AJ, Aghar-Jaffar R, Shun-Shin MJ, Francis D, Oliver N, et al. Association Between Use of Sodium-Glucose Cotransporter 2 Inhibitors, Glucagon-like Peptide 1 Agonists, and Dipeptidyl Peptidase 4 Inhibitors With All-Cause Mortality in Patients With Type 2 Diabetes: A Systematic Review and Meta-analysis. JAMA. 2018 Apr 17;319(15):1580-1591.doi: 10.1001/jama.2018.3024.

34- Verma S, Rawat S, Ho KL, Wagg CS, Zhang L, Teoh H, et al. Empagliflozin Increases Cardiac Energy Production in Diabetes: Novel Translational Insights Into the Heart Failure Benefits of SGLT2 Inhibitors. JACC Basic TransI Sci. 2018 Aug 26;3(5):575-587. doi: 10.1016/j.jacbts.2018.07.006.

35- Fitchett D, Inzucchi SE, Cannon CP, McGuire DK, Scirica BM, Johansen OE, et al. Empagliflozin Reduced Mortality and Hospitalization for Heart Failure Across the Spectrum of Cardiovascular Risk in the EMPA-REG OUTCOME Trial. Circulation. 2019 Mar 12;139(11):1384-1395. doi: 10.1161/ CIRCULATIONAHA.118.037778.

36- Rådholm K, Figtree G, Perkovic V, Solomon SD, Mahaffey KW, de Zeeuw D, et al. Canagliflozin and heart failure in type 2 diabetes mellitus. Circulation. 2018 Jul 31;138(5):458-468. doi: 10.1161/ CIRCULATIONAHA.118.034222.

37- McMurray JJV, Solomon SD, Inzucchi SE, Køber L, Kosiborod MN, Martinez FA, et al. DAPA-HF Trial Committees and Investigators. Dapagliflozin in Patients with Heart Failure and Reduced Ejection Fraction. N Engl J Med. 2019 Nov 21;381(21):1995-2008. doi: 10.1056/NEJMoa1911303.

38- Kosiborod M, Cavender MA, Fu AZ, Wilding JP, Khunti K, Holl RW, et al. Lower Risk of Heart Failure and Death in Patients Initiated on Sodium- Glucose Cotransporter-2 Inhibitors Versus Other GlucoseLowering Drugs: The CVD-REAL Study (Comparative Effectiveness of Cardiovascular Outcomes in New Users of Sodium-Glucose Cotransporter-2 Inhibitors). Circulation. 2017 Jul 18;136(3):249-259. doi: 10.1161/CIRCULATIONAHA.117.029190.

39- Kosiborod M, Lam CSP, Kohsaka S, Kim DJ, Karasik A, Shaw J, et al. Cardiovascular Events Associated With SGLT-2 Inhibitors Versus Other Glucose-Lowering Drugs: The CVD-REAL 2 Study. J Am Coll Cardiol. 2018 Jun 12;71(23):2628-2639. doi: 10.1016/j.jacc.2018.03.009.

40- Wiviott SD, Raz I, Bonaca MP, Mosenzon O, Kato ET, Cahn A, et al. Dapagliflozin and Cardiovascular Outcomes in Type 2 Diabetes. N Engl J Med 2019; 380:347-357 DOI: 10.1056/NEJMoa1812389

41- Zelniker TA, Wiviott SD, Raz I, Im K, Goodrich EL, Bonaca MP, et al. SGLT2 inhibitors for primary and secondary prevention of cardiovascular and renal outcomes in type 2 diabetes: a systematic review and meta-analysis of cardiovascular outcome trials. Lancet. 2019 Jan 5;393(10166):30.

42- Packer M, Anker SD, Butler J, Filippatos G, Ferreira JP, Pocock SJ, et al. Effect of Empagliflozin on the Clinical Stability of Patients with Heart Failure and a Reduced Ejection Fraction: The EMPERORReduced Trial. Circulation. 2021 Jan 26;143(4):326-336. doi: 10.1161/CIRCULATIONAHA.120.051783.

43- Arroyo D, Goicoechea M. Fármacos Antidiabéticos Orales e Insulinas [Internet]. Nefrol al día. 03 set. 2020. [citado 03/2021] Disponible en: https://www.nefrologiaaldia.org/330

44- Perkovic V, Jardine MJ, Neal B, Bompoint S, Heerspink HJL, Charytan DM, et al. Canagliflozin and Renal Outcomes in Type 2 Diabetes and Nephropathy. N Engl J Med. 2019 Jun 13;380(24):2295-2306. doi: 10.1056/NEJMoa1811744. 
45- Heerspink HJL, Stefánsson BV, Correa-Rotter R, Chertow GM, Greene T, Hou FF, et al. Dapagliflozin in Patients with Chronic Kidney Disease. October 8, 2020. N Engl J Med 2020; 383:1436-1446. DOI: 10.1056/NEJMoa2024816

46- ClinicalTrials.gov [Internet]. Bethesda (MD): National Library of Medicine (US). Identifier NCT03594110, EMPA-KIDNEY (The Study of Heart and Kidney Protection With Empagliflozin); 2018 July 20, [cited 2021 Feb 22]; Available from: https://www.clinicaltrials.gov/ct2/show/results/NCT03594110

\section{Aportes de cada autor al trabajo}

Zulay Andrea García Rojas: Concepción y diseño de la revisión, búsqueda bibliográfica y redacción del manuscrito.

Diana Marcela Cristancho Sierra: Búsqueda bibliográfica y redacción del manuscrito.

Amalia Verónica Peréz Papadópulos: Revisión crítica del manuscrito y redacción del manuscrito.

Gabriela Ormaechea Gorricho: Concepción y diseño de la revisión, revisión crítica del manuscrito y redacción del manuscrito. 